\title{
Citología urinaria: ¿Qué sabemos?
}

\section{Urinary Cytology: What do we know?}

\author{
Oscar Leguizamón López ${ }^{1}$ Wilfredo Donoso Donoso ${ }^{2}$ Andrés Barrios ${ }^{3}$ Rodolfo Varela ${ }^{4}$
}

1 Residente de Urología, Universidad Nacional de Colombia, Colombia
2 Urólogo, coordinador del postgrado de urología de la Universidad
Nacional de Colombia, Colombia
${ }^{3}$ Médico General, Universidad de la Sabana, Colombia
${ }^{4}$ Urólogo Oncólogo, profesor asociado de la Universidad Nacional de
Colombia, Instituto Nacional de Cancerología, Bogotá DC, Colombia

Address for correspondence Andrés Barrios, MD, Médico General, Universidad de la Sabana, Colombia (e-mail: andresbarrios207@hotmail.com).

\section{Resumen \\ Palabras Clave \\ - citologia urinaria \\ - cancer de vejiga}

El cáncer de vejiga es, en frecuencia, el segundo cáncer urológico y representa una mortalidad significativa. Es necesario entonces contar con herramientas diagnósticas efectivas que contribuyan a la toma de decisiones terapéuticas y de seguimiento. La citología urinaria es un método útil para dicho fin, pese a que es bien conocida su baja capacidad de identificación de tumores de bajo grado. Se ha demostrado cómo la citología urinaria es capaz de predecir la presencia de tumores uroteliales de alto grado que son, finalmente, aquellos que ameritan intervención pronta y ponen en riesgo la vida del paciente. Correctamente utilizada, la citología urinaria representa un apoyo fundamental en la toma de decisiones cuando el urólogo aborda el cáncer de vejiga.

Bladder cancer is in frecuency, the second urological cancer and represents significant mortality. So is necessary to have effective diagnostic tools which contribute to therapeutic decision-making and monitoring. The urinary cytology has been and still is a useful method for this purpose, although it is well known their low ability to identify low-grade tumors. It has been shown how urinary cytology can predict the presence of high-grade urothelial tumors, which are finally those who deserve strict intervention and endanger the patient's life. Properly used, urinary cytology represents a fundamental support in decision-making when the urologist deals with bladder cancer.

\section{Introducción}

Según los últimos datos de la Asociación Americana de Urología, aproximadamente 600.000 nuevos casos de cáncer de vejiga se diagnostican anualmente en los Estados Unidos, lo que condicionaría alrededor de 13.000 muertes al año por la enfermedad. ${ }^{1}$ Es el segundo cáncer urológico más frecuente, con un riesgo global de desarrollarse durante la vida de 1 en $28 .{ }^{1}$

Han sido estudiados y utilizados diversos métodos para la detección de tumores de vejiga, dentro de los cuales la citología urinaria y la cistoscopia representan los pilares del diagnóstico. Es poco probable que alguna otra estrategia diagnóstica supere los datos que pueden ser obtenidos mediante la cistoscopia, por lo que difícilmente podrá ser reemplazada. La citología urinaria, por su parte, se ha mantenido como un método diagnóstico y de seguimiento, ofreciendo alta especificidad para los tumores uroteliales de alto grado. Sería ideal que la prueba contara con la misma capacidad de identificación en tumores de bajo grado, sin embargo, en esos tumores que representan menor riesgo de progresión y recurrencia, la

\section{received}

March 8, 2018

accepted

September 24, 2018
DOI https://doi.org/

$10.1055 / \mathrm{s}-0038-1676314$.

ISSN 0120-789X.

eISSN 2027-0119.
Copyright (c) 2019, Sociedad Colombiana License terms de Urología. Publicado por Thieme Revinter Publicações Ltda., Rio de Janeiro, Brazil. Todos los derechos reservados. 
urgencia del diagnóstico puede ser menos importante por este método. Además, la alta especificidad en los tumores de alto grado contribuye a la menor realización de instrumentaciones innecesarias (cistoscopias) y a planear estrategias de tratamiento más agresivas. ${ }^{2}$

La citología urinaria sigue siendo el método con el cual se comparan potenciales estrategias nuevas de detección de cáncer de vejiga. Su alta especificidad para tumores de alto grado le confiere muy pocos casos de falsos positivos. Aunque cuenta con una baja sensibilidad para los tumores de bajo grado, se conoce que a pesar de que la probabilidad de recurrencia superficial de una lesión de bajo grado es hasta el 80\%, la progresión de ese tipo de lesión hacia una músculo-invasiva o metastásica solo representa el $15 \%$ de los casos. ${ }^{3}$ La citología urinaria puede identificar la presencia de lesiones de alto grado, incluso antes de hacerse evidentes por vía cistoscópica, con lo que se indica su uso en el seguimiento y en la rebúsqueda de lesiones uroteliales in situ en el árbol urinario superior o uretrales. ${ }^{3}$

Con respecto a los tumores de bajo grado, con el trabajo de Croskey y cols., en 2015, se observó que la sensibilidad, especificidad, valor predictivo positivo y valor predictivo negativo de la citología urinaria revisada por diferentes patólogos, cuando se comparaba con el resultado histopatológico, era del $21 \%-53 \%, 81 \%-95 \%, 71 \%$ - 90\%, y $57 \%$ - 67\%, respectivamente. El nivel de acuerdo entre los patólogos resultó ser apenas moderado. Se concluyó entonces que la citología urinaria ofrece baja sensibilidad aunque mejor especificidad para la identificación de lesiones de bajo grado, resultando en baja exactitud para su diagnóstico. ${ }^{4}$

Se han estudiado el uso de biomarcadores específicos para la identificación de tumores vesicales, dentro de los que se incluyen el análisis por imagen fluorescente, la inmunocitología de anticuerpos monoclonales, los productos de degradación de la fibrina, la medición de proteína de matriz nuclear y otros. ${ }^{5}$ Métodos con igual técnica de recolección y con la ventaja del aumento de la sensibilidad para hacer diagnóstico y detectar la recidiva de las lesiones. ${ }^{6}$

Se han comparado los resultados de dichos métodos con los conseguidos con la citología urinaria y, en general, la citología es mejor para la identificación del cáncer urotelial de alto grado y especialmente del carcinoma in situ. ${ }^{5}$ Cabe hacer énfasis en que la presencia de tumor urotelial de alto grado en la citología urinaria de un paciente con carcinoma papilar de bajo grado, sugiere lesiones no resecadas, carcinoma in situ o la presencia de carcinoma urotelial del tracto alto. La citología urinaria, entonces, aún se considera una herramienta útil en el manejo de los pacientes con tumores uroteliales. ${ }^{5}$

La mejor forma de recolectar la muestra de orina para la adecuada realización de una citología urinaria es la muestra de micción espontánea aleatoria ${ }^{7}$. Las muestras por cateterismo o por barbotaje pueden incrementar el número de células por muestra y ofrecer células mejor preservadas, pero no se recomienda realizar cateterismo de forma rutinaria para obtener muestras de orina con esa intención. En paciente con citología positiva, pero con cistoscopia negativa, se deben descartar lesiones del tracto urinario superior y CIS de vejiga; por lo que es razonable repetir la muestra de una citología positiva ${ }^{7}$.
VandenBussche y cols., demostraron en 2015 la importancia del volumen urinario enviado como muestra para citología, concluyendo que volúmenes mayores de $30 \mathrm{ml}$ ofrecen mayor probabilidad de brindar resultados positivos para lesiones de alto grado. ${ }^{8}$ No se requiere preservación de la muestra en alcohol, incluso si se amerita tiempo prolongado de almacenamiento. Usando criterios adecuados, el diagnóstico diferencial de tumor urotelial versus cambios reactivos o por regeneración por litiasis, puede casi siempre ser realizado. Además, agentes intravesicales como la Mitomicina C, pueden generar cambios característicos en las células uroteliales, pero muy raramente pueden asemejarse a cambios por carcinoma. ${ }^{9}$ Como se mencionó antes, existen otros métodos de identificación de tumores uroteliales, pero su uso de rutina aún requiere mayor refinamiento. ${ }^{9}$

Hasta hace unos años no existía una manera uniforme de reportar los resultados de una citología urinaria. Por tal motivo, el grupo del Hospital Johns Hopkins en Baltimore, Maryland (Estados Unidos), se encargó de diseñar una plantilla que estandarizara los diagnósticos citopatológicos en la orina y permitiera a los urólogos manejar de forma uniforme a sus pacientes basados en los reportes generados de las muestras y les permitiera determinar qué pacientes deben ser llevados a cistoscopia.

La plantilla diagnóstica ( - Tabla 1 ) se basó, en parte, en el sistema Bethesda para citología ginecológica. El cual se clasificó en 5 categorías posibles: negativo, carcinoma urotelial, células uroteliales atípicas, otros y muestra inadecuada; las muestras inadecuadas son raras $y$ usualmente se deben a baja celularidad o a material acelular. ${ }^{10}$

Antes de la postulación de la plantilla como un estándar, los autores evaluaron su utilidad mediante la correlación entre los hallazgos en citología y en patología quirúrgica (histológica) durante dos años (2007 - 2009). Determinaron la cantidad de pacientes con hallazgo por biopsia/RTUv de carcinoma urotelial que tenían una citología positiva para AUC-H o HGUC, máximo 6 meses previa al procedimiento. Más de la mitad de los especímenes resecados positivos para carcinoma de alto grado tenían al menos una citología previa positiva para AUC-H o HGUC. Cuando sumaron al análisis al

Tabla 1 Categorías diagnósticas del Hospital Johns Hopkins para muestras de citología del tracto urinario ${ }^{10}$

\begin{tabular}{|l|}
\hline Negativo para atipia urotelial o malignidad (NUAM) \\
\hline Carcinoma urotelial: \\
\hline Bajo grado (LGUC) \\
\hline Alto grado (HGUC) \\
\hline Células uroteliales atípicas (AUC) \\
\hline $\begin{array}{l}\text { Células uroteliales atípicas de significado indeterminado } \\
\text { (AUC-US) }\end{array}$ \\
\hline $\begin{array}{l}\text { Células uroteliales atípicas que no excluyen } \\
\text { HGUC (AUC-H) }\end{array}$ \\
\hline Otros (carcinoma escamocelular, adenocarcinoma, etc.) \\
\hline Inadecuada \\
\hline
\end{tabular}


AUC-US, el $80 \%$ de los pacientes positivos para carcinoma de alto grado en la resección tenían al menos un resultado previo positivo en la citología. De los pacientes con hallazgo citológico de células uroteliales atípicas, el $45 \%$ con AUC-US previo resultaron positivos para carcinoma de alto grado en la resección, mientras que el $71 \%$ de los pacientes con AUC-H previo fueron positivos tras la resección, mostrando una diferencia estadísticamente significativa ( $p=0.0001)$ que podría orientar a incluir la categoría AUC-US en NUAM. $^{10}$

Otro estudio determinó los hallazgos citomorfológicos observados en las citologías con AUC-H que demostraran ser más predictivos de carcinoma urotelial de alto grado. Los hallazgos morfológicos más comunes observados en AUC-H fueron, la hipercromasia, la irregularidad de los bordes nucleares, el incremento del radio núcleo-citoplasma y la anisonucleosis. De 58 pacientes que tenían citologías con diagnóstico de AUC-H, el 95\% finalmente fueron diagnosticados con carcinoma urotelial de alto grado en las resecciones. Se detectó la hipercromasia como el predictor más fuerte de carcinoma urotelial de alto grado en dichos pacientes, incluso en ausencia de los otros hallazgos descritos. Adicionalmente, los autores intentaron llevar a cabo el mismo análisis para la otra categoría citológica de células atípicas, AUC-US. En contraste con el grupo de AUC-H, en este grupo la mayoría de las citologías no contenían células atípicas con los 4 hallazgos morfológicos comunes. ${ }^{11}$

Utilizando la categoría diagnóstica del Hospital Johns Hopkins, en 2015 Wu y cols., estudiaron el diagnóstico citológico relacionado con el histológico tras la resección o biopsia. Como resultado, se encontró que más de la mitad de los pacientes (58\%) que tuvieron confirmación histológica de carcinoma urotelial de alto grado, tenían citologías previas positivas para AUC-H o HGUC; AUC-H y HGUC se asociaron con lesiones uroteliales de alto grado en el $80 \%$ y $90 \%$ de los casos respectivamente y mostraron significancia estadística cuando se compararon con AUC-US y NUAM $(p<0.05)$. Los autores concluyeron que la clasificación diagnóstica de Hospital Johns Hopkins es útil y efectiva para la identificación de pacientes con lesiones uroteliales de alto grado que requieren ser llevados a cistoscopia. ${ }^{12}$

El seguimiento después del tratamiento comienza con el examen citológico de orina cada 3 meses durante el primero o dos primeros años, dependiendo de los factores de riesgo del paciente. Los intervalos de realización de la citología se extienden en función de los hallazgos celulares y cistoscópicos anteriores y se continuará utilizando por el resto de la vida del paciente. ${ }^{10}$

La citología urinaria continúa siendo una herramienta importante en el diagnóstico y el seguimiento del cáncer urotelial, pese a la bien conocida baja sensibilidad para las lesiones de bajo grado. Tal vez la "mala fama" del examen se debe a la forma equívoca en la que se emplea. La citología urinaria cumple bien con la labor de la identificación de los tumores de alto grado, que finalmente son los que ameritan intervención con prioridad. Por otro lado, los tumores de bajo grado por sí solos no amenazan la vida del paciente, aunque tienen riesgo de convertirse en lesiones de alto grado.

\section{Referencias}

1 Hall MC, Chang SS, Dalbagni G, et al; American Urological Association. Guideline for the management of nonmuscle invasive bladder cancer (stages Ta, T1, and Tis): 2007 update. J Urol 2007;178 (06):2314-2330

2 Droller MJ. Current concepts of tumor markers in bladder cancer. Urol Clin North Am 2002;29(01):229-234

3 Barkan GA, Wojcik EM. Genitourinary cytopathology (kidney and urinary tract). Cancer Treat Res 2014;160:149-183

4 McCroskey Z, Pambuccian SE, Kleitherms S, et al. Accuracy and interobserver variability of the cytologic diagnosis of low-grade urothelial carcinoma in instrumented urinary tract cytology specimens. Am J Clin Pathol 2015;144(06):902-908

5 Rathert P. [Urinary cytology in cases of bladder cancer: a critical evaluation]. Urologe A 2003;42(07):908-911

6 Girón Rafael. Alonso Julio. Urinary cytological diagnosis: points of interest for the cytotechnologist. Rev Esp Patol 2012;45:204

7 Citarella D, Quiroga W, Fernandez F, et al. Guia de manejo en cancer vesical. Urol Colomb 2016;25:154-168

8 VandenBussche CJ, Rosenthal DL, Olson MT. Adequacy in voided urine cytology specimens: The role of volume and a repeat void upon predictive values for high-grade urothelial carcinoma. Cancer Cytopathol 2016;124(03):174-180

9 Murphy W. Current status of urinary cytology in the evaluation of bladder neoplasms. Hum Pathol 1990;21(09):886-896

10 Rosenthal D, Vandenbussche CJ, Burroughs FH, et al. The Johns Hopkins Hospital template for urologic cytology samples. Part ICreating the template. Cancer Cytopathology. Año 2013;121:15-20

11 VandenBussche CJ, Sathiyamoorthy S, Owens CL, Burroughs FH, Rosenthal DL, Guan H. The Johns Hopkins Hospital template for urologic cytology samples: parts II and III: improving the predictability of indeterminate results in urinary cytologic samples: an outcomes and cytomorphologic study. Cancer Cytopathol 2013;121(01):21-28

$12 \mathrm{Wu} \mathrm{HH}$, Redelman M, Chen S, Grignon DJ, Cramer HM. The application of the Johns Hopkins Hospital Template on urine cytology. Diagn Cytopathol 2015;43(08):593-597 